\title{
Noise Fault Diagnosis of the Refrigerator Based on Wavelet Denoising Method
}

\author{
Haijiang Kou \\ Advanced Technology Department, Hisense (Shandong) \\ Refrigerator Co. , Ltd \\ Qingdao, China. \\ khj136@163.com \\ kouhaijiang@hisense.com
}

\begin{abstract}
Noise reduction technology of the refrigerator has become one of the core competitiveness and low noise gives users a comfortable experience. A new experimental investigation for noise source diagnosis of the refrigerator is conducted based on wavelet denoising method. And wavelet denoising is compared with conventional method of correction. It is shown that wavelet denoising can separate high or low frequency part and high or low frequency noise of test signals. It is thus capable of revealing fault information when removing noise signals. And the sound power of the improving refrigerator is decreased by $1.3 \mathrm{~dB}$ through improving vacuum degree of pipeline of the fault refrigerator. This indicates that wavelet denoising method is effective.
\end{abstract}

Keywords-refrigerator, wavelet denoising, fault diagnosis, data preprocessing

\section{INTRODUCTION}

In the development process of the refrigerator, noise problem is paid more attention by users. Solutions for reducing noise are urgently required and many studies have been conducted to reduce the noise of the refrigerator. Han and Kim et al. [1] identified irregular refrigerant noise through the observation of the relationship between the flow pattern and the refrigerant-induced noise. Then, Kim [2] performed experiments using R600a refrigerant and refrigerant-supply equipment designed for flow visualization and flow regime transition. Lee et al. [3] predicted the internal BPF noise using the modeled source $\mathrm{s}$ in combination with the boundary element method. D.G. Lee et al. [4] defined the generation mechanism of this abnormal noise in the form of a ticking sound inside of a refrigerator which was generated by the relative distortion and slip between the inner structures. While experimental data in these studies is always contain random non-stationary noise, it is more difficult to solve the problem of the refrigerator noise due to fault information being submerged in the noise.

The signal denoising process using wavelet transform is an important area of research in structural and machine fault diagnosis [5-8]. Wavelet analysis which is proven to be useful tools for suppressing additive noise and restoring the signals overcomes the drawbacks of other techniques by means of analytical functions that are local in both time and frequency

\author{
Jiaojiao $\mathrm{Du}$ \\ School of Mechanical \& Electrical Engineering, Qingdao \\ Binhai University \\ Qingdao, Shandong, China. \\ dujiao1769@163.com
}

[9-10]. Therefore, it is also suitable to use wavelet denoising method to carry out noise fault diagnosis of the refrigerator.

In this paper, a new experimental investigation for noise source diagnosis of the refrigerator is conducted based on wavelet denoising method. And wavelet denoising is compared with conventional method of correction in order to reveal the capabilities of the present method in modification of non-stationary noisy signals. In addition, vacuum degree of pipeline of the fault refrigerator is improved to demonstrate the potential fault and the validity of wavelet denoising.

\section{The WaVelet Denoising Method}

Wavelet transform can make signals to be decomposed in different scales to reflect the different feature of the noise and the useful signal. Threshold wavelet transform method for signal denoising is shown in figure 1.

Contaminated signal function is defined as

$$
f(t)=s(t)+\sigma n(t)
$$

Where $f$ is original signal which has mixed noise signals, $s$ is the fault signal function, $n$ is noise signal function, $\sigma$ is noise intensity.

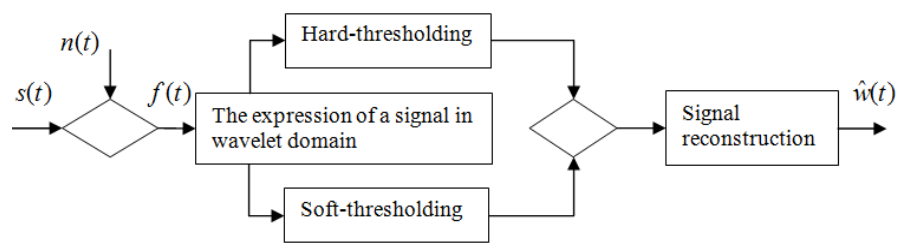

Fig. 1. The principle diagram of the wavelet denoising

The main steps applying the wavelet decomposition and reconstruction method in signal denoising are based on hardthresholding and soft-thresholding. The main processes are as follows: Firstly, decompose signal $f$ into approximate coefficients and detailed coefficients in a certain scales. In this way, we can obtain wavelet coefficients. The wavelet denoising is achieved via thresholding to transform wavelet coefficients. And then, reconstructing signal through a new coefficient which enables fault detection and diagnosis. For 
soft- thresholding, wavelet coefficients is zero when their absolute value less than or equal to the threshold value. And wavelet coefficients become the difference between wavelet coefficients and threshold value. Hard-thresholding only makes wavelet coefficients to be zero when their absolute value less than or equal to the threshold value. Because signal information is smoother with soft-thresholding method, this paper uses this method for signal denoising.

\section{EXPERIMENTAL RESULTS}

In order to find noise source of the refrigerator, a set of the fault diagnosis experiments were performed as shown in Fig. 2(a). Test signals were mainly measured by microphones. And sound power in this paper was calculated according to the standard of IEC-60704. Fig. 2(b) shows the results of measured original non-stationary signal. Then Fig. 3 gives signal denoising by band-pass filter and wavelet method. It can be directly seen that most of the noise is removed by the wavelet transformation de-noise method and band-pass filter. And the difference of both methods is not obvious.

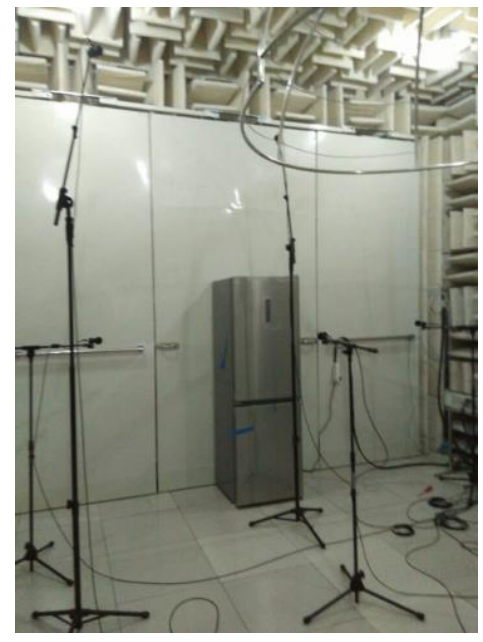

(a)

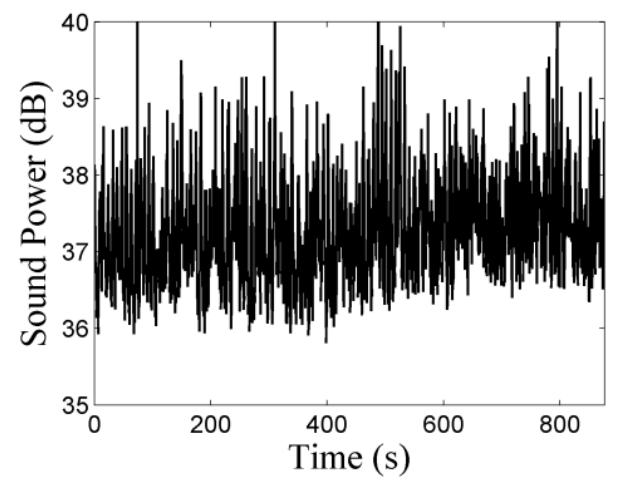

(b)

Fig. 2. The fault diagnosis experiments: (a) distribution of the sensor; (b) the sound power in different time intervals

\section{NOISE FAUlt Diagnosis}

In order to find noise source of the refrigerator, measured non-stationary signal denoising by both methods is compared with the signal from no fault refrigerator, as shown in Fig. 4. There are three frequencies of failure: $400 \mathrm{~Hz}, 1250 \mathrm{~Hz}$, and $8000 \mathrm{~Hz}$ using band-pass filter in Fig. 4(a). Therefore, it is difficult to find the noise source. By contrast, it can be obviously found fault frequency $1250 \mathrm{~Hz}$ components using wavelet denoising in Fig. 4(b). Band-pass filter cannot effectively separate high or low frequency part and high or low frequency noise of test signals. Because non-stationary signals have well reserves of the useful signal peak and saltation parts by wavelet denoising which is suitable for nonstationary signals de-noise.

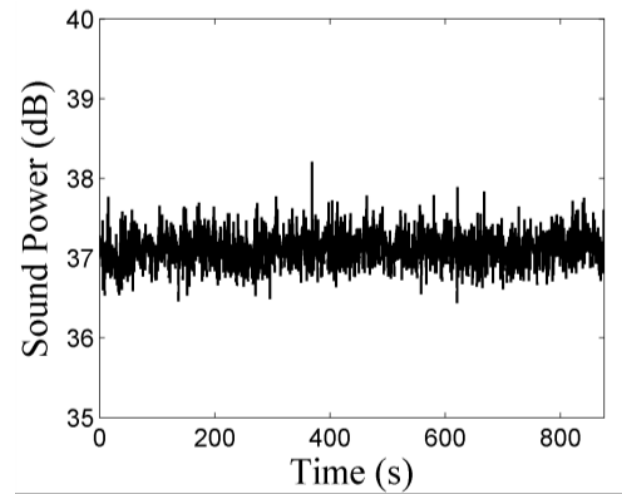

(a)

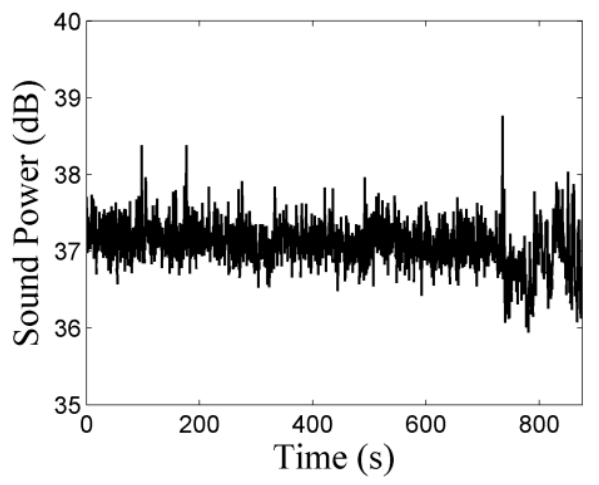

(b)

Fig. 3. Measured non-stationary signal denoising in different time intervals for: (a) wavelet denoising; (b) band-pass filter

The frequency component $1250 \mathrm{~Hz}$ is usually from refrigerant flow noise. By improving vacuum degree of pipeline of the fault refrigerator, sound power results are obtained in table 1. It can be found that the sound power of the improving refrigerator is decreased by $1.3 \mathrm{~dB}$. These results indicate that wavelet denoising method is effective. 
TABLE I. THE COMPARISON OF SOUND POWER BETWEEN NO FAULT REFRIGERATOR AND FAULT REFRIGERATOR

\begin{tabular}{|c|c|c|c|}
\hline & \multirow{2}{*}{$\begin{array}{l}\text { No fault } \\
\text { refrigerator }\end{array}$} & \multicolumn{2}{|c|}{ Fault refrigerator } \\
\hline & & $\begin{array}{c}\text { Before } \\
\text { improvement }\end{array}$ & $\begin{array}{c}\text { After } \\
\text { improvement }\end{array}$ \\
\hline $\begin{array}{c}\text { Sound } \\
\text { Power }(\mathrm{dB})\end{array}$ & 36.8 & 37.8 & 36.5 \\
\hline
\end{tabular}

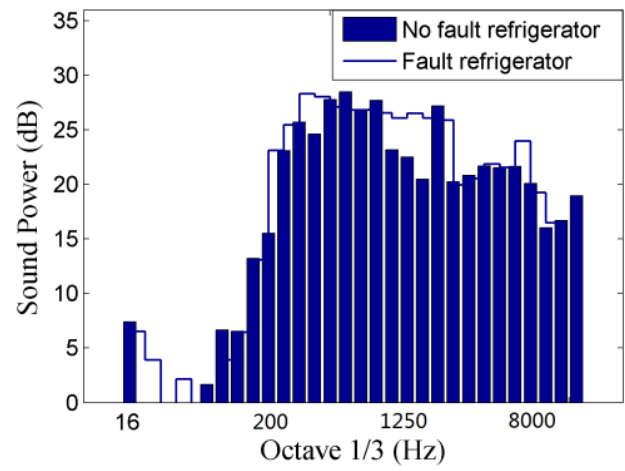

(a)

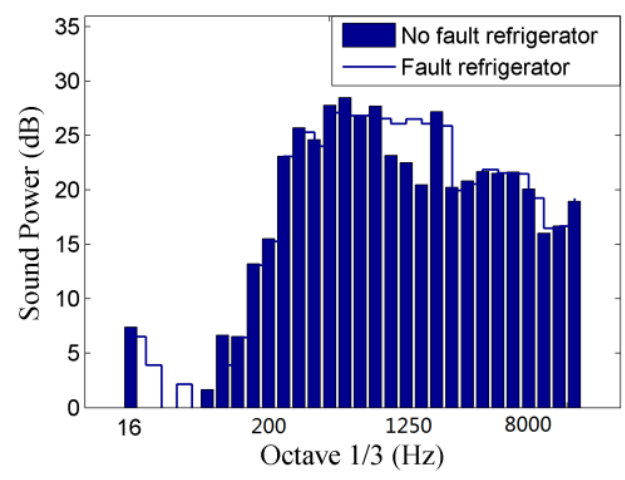

(b)

Fig. 4. Measured signals amplitude spectrum diagrams: (a) band-pass filter; (b) wavelet denoising

\section{CONCLUSIONS}

Based on wavelet denoising method, a new experimental investigation for noise source diagnosis of the refrigerator is conducted. Compared with conventional modification method of non-stationary noisy signals, wavelet denoising can separate high or low frequency part and high or low frequency noise of test signals. It is thus capable of revealing fault information when removing noise signals. By improving vacuum degree of pipeline of the fault refrigerator, it can be found that the sound power of the improving refrigerator is decreased by $1.3 \mathrm{~dB}$. This indicates that wavelet denoising method is effective.

\section{ACKNOWLEDGMENT}

This research was supported by National Natural Science Foundation of China under Project No: 50775028 and
Shandong Higher School Superior Discipline Talent Team Development Program of China.

\section{REFERENCES}

[1] H. S. Han, W. B. Jeong, M. S. Kim, T. H. Kim. "Analysis of the root causes of refrigerant-induced noise in refrigerators," J. Mech. Sci. Technol, vol. 23, pp. 3245-3256, December 2009.

[2] M. S. Kim, W. B. Jeong, H. S. Han. "Development of noise pattern map for predicting refrigerant-induced noise in refrigerators," J. Mech. Sci. Technol, vol. 28, pp. 3499-3510, September 2014.

[3] S. Lee, S. Heo, C. Cheong. "Prediction and reduction of internal bladepassing frequency noise of the centrifugal fan in a refrigerator," Int. J. Refrig, vol. 33, pp. 1129-1141, September 2010.

[4] D. G. Lee, J.H. Park, K. H. Park, B. K. Ha, H. S. Kim, S. H. Park. "Investigation of the Frictional Impulse Induced Abnormal Noise in a Refrigerator,” Int. J. Precis. Eng. Man, vol. 14, pp. 559-566, April 2013.

[5] C. Mishra, A. K Samantaray, G Chakraborty. "Rolling element bearing defect diagnosis under variable speed operation through angle synchronous averaging of wavelet de-noised estimate," Mech. Syst. Signal. Pr, vol. 72-73, pp. 206-222, May 2016.

[6] Z. Liu, Z. He, W. Guo. "A hybrid fault diagnosis method based on second generation wavelet de-noising and local mean decomposition for rotating machinery”. ISA. Trans, vol. 61, pp. 211-220, March 2016.

[7] X. Meng, R. Xie, C. Li. "An NMR log echo data de-noising method based on the wavelet packet threshold algorithm". J. Geophys. Eng, vol. 12, pp. 956-968, December 2015.

[8] S. W. Chen, Y. H. Chen. "Hardware Design and Implementation of a Wavelet De-Noising Procedure for Medical Signal Preprocessing". Sensors, vol. 15, pp. 26396-26414, October 2015.

[9] J. Rafiee, P.W. Tse, A. Harifi, M.H. Sadeghi. "A novel technique for selecting mother wavelet function using an intelligent fault diagnosis system," Expert. Syst. Appl. vol. 36, pp. 4862-4875, April 2009.

[10] B. Tang, W. Liu, T. Song. "Wind turbine fault diagnosis based on Morlet wavelet transformation and Wigner-Ville distribution," Renew. Energ. vol. 35, pp. 2862-2866, December 2010. 